\title{
Estrutura e suporte familiar como fatores de risco de stress infantil
}

\author{
Family pillar and structure as risk \\ factors to child stress
}

\author{
Mônica Augusta MOMBELLI' \\ Jaquilene Barreto da COSTA \\ Sonia Silva MARCON ${ }^{2}$ \\ Cynthia Borges de MOURA $^{3}$
}

\begin{abstract}
Resumo
Este estudo investigou a relação entre a percepção acerca do suporte familiar e o stress infantil em 30 crianças, de ambos os sexos, de 7 a 12 anos de idade, participantes do Projeto Família em Ação, promovido pela Secretaria Municipal de Saúde do Município de Matelândia. Trata-se de um estudo descritivo exploratório, que utilizou como instrumento para coleta de dados o Inventário de Percepção do Suporte Familiar e a Escala de Estresse Infantil, aplicados individualmente. Os resultados indicaram que 60\% das crianças apresentaram baixo suporte familiar e stress; dessas, 50\% moravam com suas famílias intactas. A sintomatologia de stress predominante é psicológica, com 50\%, indicando a vulnerabilidade da criança para a inadaptação psicossocial. Discute-se, dessa forma, a importância de trabalhos de prevenção e fortalecimento das redes de apoio social nas quais a família esteja inclusa, visando o bem-estar psicológico das crianças.
\end{abstract}

Unitermos: Stress psicológico. Relações familiares. Saúde mental.

\begin{abstract}
This study investigated the relationship between the perceptions concerning family support and child stress in 30 children of both sexes, between the ages of 7 and 12, who were participating in the "Family-in-Action Project" promoted by the Department of Health in the city of Matelândia, in the state of Paraná. It is a descriptive-exploratory study that used, as a data collection tool, the Inventory of Perception of Family Support and the Child-stress Scale, both applied individually. Results indicated that $60 \%$ of the children presented low family support and stress, and of these 50\% lived in families that were still intact. The predominant symptoms of stress were psychological, affecting 50\%, which indicates child vulnerability to psycho-social non-adaptation. Thus, the importance of preventive work and the strengthening of the social-support network is discussed, where families are inclusive, directed towards the psychological well-being of ourchildren.
\end{abstract}

Uniterms: Mental health. Family relations. Psychological stress.

\section{प्रण}

1 Universidade Estadual de Maringá, Centro de Ciências da Saúde, Programa de Pós-Graduação em Ciências da Saúde. Av. Colombo, 5790, Bloco 111, Sala 6, 87020-900, Maringá, PR, Brasil. Correspondência para/Correspondence to: M.A. MOMBELLI. E-mails: <monicamombelli_2004@hotmail.com>; $<$ monicamombelli@pop.com.br>.

2 Universidade Estadual de Maringá, Núcleo de Estudo, Pesquisa, Assistência e Apoio à Família. Maringá, PR, Brasil.

3 Universidade Estadual do Oeste do Paraná, Centro de Educação e Letras, Curso de Enfermagem. Maringá, PR, Brasil. 
A família desempenha um significativo papel socializador, apesar das grandes modificações que esse grupo social vem sofrendo nas últimas décadas. As rápidas mudanças sociais que estão ocorrendo no núcleo das famílias ocasionam transformações na estrutura e na sua função, nas práticas educativas e nos valores coletivos e individuais de cada um de seus membros (M.N. Baptista, A.S.D. Baptista \& Dias, 2001).

As famílias, em seu modo próprio e singular de viver, podem experienciar, em seu cenário de relacionamento interpessoal, situações estressantes, como mudanças de vida ou eventos vitais, que podem desencadear alterações na saúde; nesse momento, a obtenção de suporte social/familiar desempenha um papel de destaque. Dessa forma, a família como uma unidade de análise apresenta características importantes para o estudo da relação entre o suporte familiar e o stress infantil.

Dentre as definições do que vem a ser suporte social, existe um consenso sobre o papel da família como fonte suportiva. O suporte social deve ser analisado de forma ampla a fim de que se considerem todas as relações significativas. O papel dos amigos e da comunidade deve ser considerado como importante provedor de suporte ao indivíduo, contudo a família assume um papel de destaque no adequado suporte oferecido (Miller \& Jeff-Darlington, 2002).

O suporte familiar é considerado um dos fatores de maior relevância, por ser uma instância mediadora entre o indivíduo e a sociedade, e ter como algumas de suas funções reunir e fornecer recursos para a satisfação das necessidades básicas, além de funcionar como um amortecedor de impactos sociais na vida de seus integrantes (Baptista, 2004; Carvalho \& Almeida, 2003).

O ser humano, quando tem suas expectativas satisfeitas pelo suporte social e familiar, apresenta uma menor predisposição a desordens mentais, assim como autoestima elevada, quando comparado com o que não tem suas necessidades satisfeitas (Lakey \& Scoboria, 2005). Além de o suporte familiar atenuar cotidianamente os eventos estressores na vida do indivíduo, ele se constitui um fator de proteção diante de condições adversas (Arpini \& Quintana, 2003; Carvalho \& Almeida, 2003).

A criança, tal como o adulto, está suscetível ao 328 stress e a situações estressantes excessivas ou prolon- gadas que podem gerar importantes efeitos psicopatológicos (Egeland \& Kreutzer, 1991). Em 1936, o fisiologista canadense Hans Selye introduziu o termo stress no campo da saúde para designar a resposta geral e inespecífica do organismo a um estressor ou a uma situação estressante. Posteriormente, o termo passou a ser utilizado para designar tanto essa resposta do organismo como a situação que desencadeia seus efeitos (Labrador \& Crespo, 1994).

Os sintomas do stress infantil, assim como no adulto, podem envolver reações que englobam alterações psicológicas e físicas no organismo, provenientes de fontes internas e externas. A resposta ao stress é resultado da interação entre as características da pessoa e as demandas do meio, ou seja, as discrepâncias entre os meios externo e interno e a percepção do indivíduo quanto à sua capacidade de resposta (Lipp, Souza, Romano \& Covolan, 1991).

As reações psicológicas e físicas geradas pelo stress podem influenciar também a relação entre os pais e seus filhos. Com o objetivo de cumprir o papel de agentes de socialização dos filhos, os pais fazem uso de diversas estratégias e técnicas para orientar seus comportamentos. Pais estressados tendem a utilizar-se de práticas educativas negativas, que se tornam fontes de stress para a criança (Gomide, 2003).

No Brasil, os estudos sobre stress infantil teve início na década de 1970, em São Paulo, e demonstraram que muitas características do stress adulto eram semelhantes às do stress infantil (Lipp, 2004). Alguns estudos com crianças em idade escolar têm demonstrado uma alta prevalência de stress, variando entre 30\% a 60\% (Sbaraini \& Schermann, 2008; Vilela, 1995; Tanganelli \& Lipp, 1998). Durante o desenvolvimento intelectual, emocional e afetivo, a criança encontra-se diante de inúmeras situações geradoras de tensão e, na maioria das vezes, não tem ainda habilidade para lidar com tais eventos. Porém, nem todas as crianças submetidas ao mesmo nível de tensão desenvolvem sintomatologia de stress (Bugental \& Johnston, 2000; Lipp, 2000). Os fatores de maior ou menor vulnerabilidade ao stress na infância são diretamente influenciados pelas diversas formas de apoio social que a criança recebe, principalmente o suporte oferecido pela família.

Sugere-se, dessa forma, uma coerência teórica e prática na hipótese central deste estudo: a percepção 
de um baixo suporte familiar pode ser considerada um importante preditor de transtornos afetivos em crianças, incluindo os advindos do stress, pois a avaliação do suporte recebido influencia tanto a forma como a criança avalia a si mesma e as informações provindas do meio externo, quanto a forma como reage a elas.

Destarte, o objetivo deste estudo foi investigar a relação entre a percepção da criança acerca do suporte familiar e o nível de stress que ela apresenta.

\section{Método}

\section{Participantes}

Trata-se de um estudo descritivo-exploratório realizado na cidade de Matelândia (PR), município com 15404 habitantes (Instituto Brasileiro de Geografia e Estatística, 2007), localizado na Região Oeste do Paraná. Foram informantes do estudo todas as crianças que, em 2007, frequentavam grupos terapêuticos integrantes do projeto "Família em ação", coordenado por uma psicóloga e desenvolvido com uma das quatro equipes do Programa Saúde da Família (PSF), existente no município.

Os grupos terapêuticos tiveram início devido a sucessivos encaminhamentos, ao setor de psicologia da unidade de saúde estudada, realizados por médicos e professores motivados por queixas relacionadas a dificuldades na dinâmica familiar. Assim, o elevado número de crianças com queixas específicas fez com que o setor de psicologia implantasse um trabalho de grupo nessa área. Foram constituídos três grupos terapêuticos - com duração de duas horas semanais -, cada um dos quais composto por 10 crianças. Nesse período, eram realizadas dinâmicas de grupo baseadas nos pressupostos da abordagem comportamental, a fim de se ensinarem e de se fortalecerem as habilidades comportamentais da criança.

\section{Instrumentos}

A coleta de dados foi realizada no período de setembro a novembro de 2007 por meio de entrevista estruturada com aplicação de três instrumentos: 1) ficha de identificação da criança, 2) Inventário de Percepção do Suporte Familiar (IPSF) e 3) Escala de Estresse Infantil (ESI).
O IPSF é constituído por 43 itens em uma escala do tipo Likert de três pontos. Com esse instrumento, avalia-se como o indivíduo percebe sua relação com a família nos aspectos inadaptação familiar, afetividade familiar e consistência familiar (Baptista, 2005). A ESI contém 35 itens em uma escala tipo Likert de 5 pontos e é utilizada para avaliação do stress infantil e verificação do tipo de sintomatologia apresentada pela criança (sintomas físicos, psicológicos, psicológicos com componente depressivo e psicofisiológicos) (Lipp \& Lucarelli, 1998).

\section{Procedimentos}

A ficha de identificação da criança foi preenchida pelo seu responsável por ocasião da sua inclusão no grupo, e o IPSF e a ESI foram aplicados individualmente, no mesmo local onde os encontros eram realizados, após o término das sessões. O tempo médio de aplicação foi de aproximadamente trinta minutos para cada instrumento, e foram necessários dois dias para o preenchimento dos dois instrumentos.

Para análise e correção do IPSF e ESI, utilizaram-se os critérios padronizados. As informações obtidas com os responsáveis sobre a criança e os resultados do IPSF e da ESI foram registrados e armazenados em um banco de dados do Excel, e utilizou-se para análise descritiva o pacote computacional Statistic 8.0, e para cálculo do Teste Exato de Fischer, o programa (SAS) 9.1 Statistical Analisys System. Foi estabelecido um nível de significância mínimo de 5\%.

A partir dessa análise, os dados do perfil sociodemográfico e os fatores foram comparados quanto à percepção do suporte familiar e à presença ou não de stress. Os resultados estão apresentados em forma de tabelas em frequência absoluta e relativa.

O estudo obedeceu aos preceitos éticos disciplinados pela Resolução 196/96 do Conselho Nacional de Saúde (Brasil, 1997), que regulamenta pesquisa com seres humanos. O projeto de pesquisa foi aprovado pelo Comitê de Ética e Pesquisa com Seres Humanos da Universidade Estadual de Maringá (UEM) (Parecer no 383.2007). Todos os responsáveis assinaram o termo de consentimento livre e esclarecido em duas vias. 


\section{Resultados}

Participaram do estudo 30 crianças e seus respectivos responsáveis. As idades das crianças variaram entre 7 e 12 anos, ficando a idade média em 9,1 anos. Em sua maioria, as crianças eram do sexo feminino $(83,3 \%)$ e estavam cursando o ensino fundamental (76,7\%). Quanto à estrutura familiar, metade delas morava com os pais, 30,0\% com a mãe, 13,3\% em famílias reconstituídas e 6,7\% com os avôs (Tabela 1).

O IPSF utilizado para pesquisa mostrou que 70,0\% das crianças percebiam o suporte familiar como baixo, e os demais, como médio; nenhuma das crianças, portanto, percebia alto suporte familiar.

Das crianças que apresentaram baixo suporte familiar, 17 são meninas (81,0\%). Houve maior predominância de baixo suporte familiar em crianças com 9 anos ou mais (85,8\%), que cursavam de $1^{\text {a }}$ a $4^{\text {a }}$ série (76,2\%), tinham de 3 a 4 irmãos (55,0\%) e moravam com os pais (52,4\%). A análise estatística revelou associação significativa entre a percepção de suporte familiar pela criança e o número de irmãos ( $p=0,0037)$. Não foi observada associação significativa entre a percepção de suporte familiar e as variáveis individuais analisadas, incluindo sexo, faixa etária, escolaridade e estrutura familiar (Tabela 1).

Das 30 crianças avaliadas, a maioria (73,3\%) apresentava indicativos de stress infantil com sintomatologia psicológica. Dessas, 50,0\% apresentavam sintomas psicológicos; nas demais, essa sintomatologia vinha acompanhada de sintomas físicos, sintomas psicológicos com componente depressivo e/ou sintomas psicofisiológicos. Em apenas 9,1\% das crianças, todos os componentes da sintomatologia do stress estiveram presentes. Os principais sintomas psicológicos foram: ansiedade, pânico, tensão, angústia, insônia, alienação, dificuldades interpessoais, dúvida quanto a si própria, preocupação excessiva, inabilidade para relaxar, tédio, ira, depressão e hipersensibilidade emotiva.

A distribuição das crianças quanto às características sociodemográficas e à presença ou não de stress infantil pode ser observada na (Tabela 2). A maioria das

Tabela 1. Distribuição da percepção do suporte familiar nos participantes da pesquisa $(n=30)$ e valores de $p$, segundo características sociodemográficas. Matelândia (PR), 2007.

\begin{tabular}{|c|c|c|c|c|c|c|}
\hline \multirow{3}{*}{ Variáveis } & \multirow{3}{*}{ Total $(n=30)$} & \multicolumn{4}{|c|}{ Suporte familiar } & \multirow{3}{*}{$p$} \\
\hline & & \multicolumn{2}{|c|}{ Baixo } & \multicolumn{2}{|c|}{ Médio } & \\
\hline & & $n$ & $\%$ & $n$ & $\%$ & \\
\hline \multicolumn{7}{|l|}{ Sexo } \\
\hline Feminino & 25 & 17 & 81,0 & 8 & 88,9 & \multirow{2}{*}{1,0000} \\
\hline Masculino & 5 & 4 & 19,0 & 1 & 11,1 & \\
\hline \multicolumn{7}{|l|}{ Faixa etária } \\
\hline 7 a 8 anos & 4 & 3 & 14,2 & 1 & 11,1 & \multirow{3}{*}{0,6411} \\
\hline 9 a 10 anos & 15 & 9 & 42,9 & 6 & 66,7 & \\
\hline$>10$ anos & 11 & 9 & 42,9 & 2 & 22,2 & \\
\hline \multicolumn{7}{|l|}{ Escolaridade } \\
\hline 1a a 4a série & 23 & 16 & 76,2 & 7 & 77,8 & \multirow{2}{*}{1,0000} \\
\hline $5^{\mathrm{a}}$ a $8^{\mathrm{a}}$ série & 7 & 5 & 23,8 & 2 & 22,2 & \\
\hline \multicolumn{7}{|l|}{ Número de irmãos } \\
\hline $1 \mathrm{a} 2$ & 9 & 2 & 10,0 & 7 & 70,0 & \multirow{3}{*}{0,0037} \\
\hline 3 a 4 & 13 & 11 & 55,0 & 2 & 20,0 & \\
\hline 5 a 6 & 8 & 7 & 35,0 & 1 & 10,0 & \\
\hline \multicolumn{7}{|l|}{ Estrutura familiar } \\
\hline Pai e mãe & 15 & 11 & 52,4 & 4 & 44,5 & \multirow{4}{*}{0,7545} \\
\hline Família reconstituída & 4 & 2 & 9,5 & 2 & 22,2 & \\
\hline Avó & 2 & 2 & 9,5 & - & - & \\
\hline Mãe & 9 & 6 & 28,6 & 3 & 33,3 & \\
\hline
\end{tabular}

330

p: Teste Exato de Fisher. 
Tabela 2. Distribuição da presença de stress nos participantes da pesquisa $(n=30)$ e valores de $p$, segundo características sociodemográficas. Matelândia (PR), 2007.

\begin{tabular}{|c|c|c|c|c|c|c|}
\hline \multirow{3}{*}{ Variáveis } & \multirow{3}{*}{ Total $(n=30)$} & \multicolumn{4}{|c|}{ Stress infantil } & \multirow{3}{*}{$p$} \\
\hline & & \multicolumn{2}{|c|}{ Sim } & \multicolumn{2}{|c|}{ Não } & \\
\hline & & $n$ & $\%$ & $\mathrm{n}$ & $\%$ & \\
\hline \multicolumn{7}{|l|}{ Sexo } \\
\hline Feminino & 25 & 19 & 86,4 & 6 & 75,0 & \multirow{2}{*}{0,5894} \\
\hline Masculino & 5 & 3 & 13,6 & 2 & 25,0 & \\
\hline \multicolumn{7}{|l|}{ Faixa etária } \\
\hline 7 a 8 anos & 4 & 4 & 18,2 & & - & \multirow{3}{*}{0,0748} \\
\hline 9 a 10 anos & 15 & 8 & 36,4 & 7 & 87,5 & \\
\hline$>10$ anos & 11 & 10 & 45,4 & 1 & 12,5 & \\
\hline \multicolumn{7}{|l|}{ Escolaridade } \\
\hline 1a a $4^{a}$ série & 23 & 13 & 68,4 & 10 & 90,9 & \multirow{2}{*}{0,2146} \\
\hline 5a a 8a série & 7 & 6 & 31,6 & 1 & 9,1 & \\
\hline \multicolumn{7}{|l|}{ Número de irmãos } \\
\hline 1 a 2 & 9 & 4 & 18,2 & 5 & 62,5 & \multirow{3}{*}{0,0264} \\
\hline 3 a 4 & 13 & 10 & 45,4 & 3 & 37,5 & \\
\hline $5 a 6$ & 8 & 8 & 36,4 & - & - & \\
\hline \multicolumn{7}{|l|}{ Estrutura familiar } \\
\hline Pai e mãe & 15 & 10 & 47,6 & 5 & 55,6 & \multirow{4}{*}{0,6584} \\
\hline Família reconstituída & 4 & 2 & 9,5 & 2 & 22,2 & \\
\hline Avó & 2 & 2 & 9,5 & - & - & \\
\hline Mãe & 9 & 7 & 33,4 & 2 & 22,2 & \\
\hline
\end{tabular}

$p$ : Teste Exato de Fisher.

crianças que apresentaram stress era de meninas (86,4\%). Houve uma prevalência de stress nas crianças maiores de 10 anos (45,5\%), que frequentavam da $1^{\text {a à }} 4^{\circ}$ série $(68,4 \%)$, tinham de 3 a 4 irmãos $(45,4 \%)$ e moravam com os pais $(47,6 \%)$.

A análise estatística revelou associação significativa entre o stress e o número de irmãos $(p=0,0264)$. Não foi observada associação significativa entre a presença de sintomas de stress e as variáveis individuais estudadas.

Ao se investigar a relação entre a percepção da criança acerca do suporte familiar e a presença de stress infantil (Tabela 3), constatou-se que a maioria das crianças $(60,0 \%)$ apresentou baixo suporte familiar e stress, reforçando a tese de que baixos níveis de suporte familiar estão associados à presença de sintomas de stress na criança. Observou-se que quanto maiores os níveis de suporte familiar, menores os sintomas de stress, chegando-se à sua ausência.

No que se refere à estrutura familiar (Tabela 4), os resultados mostraram que mesmo as crianças que viviam com os pais apresentaram baixo suporte familiar associado a sintomas de stress, sugerindo que só a convivência na família intacta não protege a criança de alterações emocionais.

\section{Discussão}

Os grupos terapêuticos foram iniciados devido aos sucessivos encaminhamentos realizados por médicos e professores destinados ao setor de psicologia da Secretaria Municipal de Saúde do município de Matelândia (PR), devido a queixas relacionadas a dificuldades

Tabela 3. Distribuição dos sujeitos quanto à relação entre stress e percepção de suporte familiar. Matelândia (PR), 2007.

\begin{tabular}{lcc}
\hline Variáveis & $\mathrm{n}=30$ & $\%$ \\
\hline Baixo suporte familiar e stress infantil & 18 & 60,0 \\
Médio suporte familiar e stress infantil & 4 & 13,3 \\
Baixo suporte familiar e ausência de stress infantil & 3 & 10,0 \\
Médio suporte familiar e ausência de stress infantil & 5 & 16,7 \\
\hline Total & 30 & 100,0 \\
\hline
\end{tabular}


Tabela 4. Relação entre estrutura familiar, suporte familiar e stress infantil. Matelândia (PR), 2007.

\begin{tabular}{|c|c|c|c|c|c|c|c|c|c|}
\hline \multirow{2}{*}{ Estrutura familiar } & \multicolumn{2}{|c|}{ Baixo suporte/stress } & \multicolumn{2}{|c|}{ Médio suporte/stress } & \multicolumn{2}{|c|}{$\begin{array}{c}\text { Baixo suporte/ausência } \\
\text { stress }\end{array}$} & \multicolumn{2}{|c|}{$\begin{array}{c}\text { Médio suporte/ausência } \\
\text { stress }\end{array}$} & \multirow{2}{*}{$p$} \\
\hline & $n$ & $\%$ & $n$ & $\%$ & $n$ & $\%$ & $\mathrm{n}$ & $\%$ & \\
\hline Pai e mãe (no 15) & 9 & 60,00 & 1 & 6,67 & 2 & 13,33 & 3 & 20,00 & \multirow{4}{*}{0,8747} \\
\hline Família reconstituída ( $n^{\circ} 4$ ) & 1 & 25,00 & 1 & 25,00 & 1 & 25,00 & 1 & 25,00 & \\
\hline Avó (n’ 2) & 2 & 100,00 & - & - & - & - & - & - & \\
\hline Mãe (n॰ 9) & 5 & 55,56 & 2 & 22,22 & 1 & 11,11 & 1 & 11,11 & \\
\hline
\end{tabular}

p: Teste Exato de Fisher.

na escola e na dinâmica familiar. As famílias que buscam auxílio externo nos recursos oferecidos pela comunidade para promover o desenvolvimento integral de seus filhos podem cooperar efetivamente para melhores resultados, inclusive em condições desfavoráveis (Leventhal \& Brooks-Gunn, 2000).

Observou-se no estudo que a maioria das crianças estava cursando o primeiro ciclo do ensino fundamental e que uma grande proporção delas frequentava séries incompatíveis com sua idade. Em estudo desenvolvido com 287 crianças de ambos os sexos, com idade entre 11 e 14 anos, de uma escola pública, foi observado que práticas parentais, como afetividade, consistência e disciplina sem prática punitiva, estão associadas a um desenvolvimento sadio dos indivíduos. Observou-se também que indivíduos que manifestam a percepção do suporte familiar como satisfatória tendem a apresentar menos comportamentos inadequados, como, por exemplo, envolvimento com substâncias químicas (Saric, Rijavec \& Zganec, 2001).

Os dados de escolaridade acima descritos corroboram os de estudos que utilizaram o mesmo instrumento para avaliar o stress de alunos de $1^{\text {a a }} 4^{\mathrm{a}}$ série, com e sem dificuldades escolares. Observaram-se altos índices de sintomas de stress relacionados com desempenho escolar fraco (Tanganelli \& Lipp, 1998; Vilela, 1995).

O tipo de estrutura familiar parece não ter interferido na percepção do suporte e também na identificação de sintomas de stress. O conceito de família vem se modificando de forma gradativa, devido à coexistência de diferentes arranjos familiares. Por estrutura familiar, entende-se a quantidade de pessoas que moram na casa e suas respectivas funções, o fato de os progenitores estarem vivos ou terem falecido, a condição de estarem divorciados ou separados ou de dividir moradia com outros parceiros, dentre outras características (Baptista et al., 2001).
As modificações ocorridas na estrutura da família podem ser observadas, por exemplo, na transição do modelo nuclear intacto (pai + mãe + filhos) para a família descasada (mãe + filhos ou pai + filhos) e, posteriormente, recasada (pai + esposa/madrasta + filhos; mãe + esposo/padrasto + filhos). A passagem de um modelo a outro requer dos membros da família uma adaptação às mudanças de relacionamento, aos papéis e à estrutura familiar, assim como às demandas do mundo externo. Apesar do incremento da complexidade das relações familiares, o processo de reestruturação da família reconstituída não será necessariamente desencadeador de conflitos. A plasticidade das relações no núcleo familiar pode gerar uma infinidade de recursos promotores de saúde (Wagner, Ribeiro, Arteche \& Bornholdt, 1999).

Pode-se inferir, dessa forma, que um funcionamento familiar adequado - em que estejam presentes a coesão familiar, boa comunicação entre seus membros, adaptabilidade da família a mudanças, bom vínculo emocional - constitui fator protetor contra o aparecimento de distúrbios emocionais em crianças, independentemente da estrutura familiar estabelecida.

No que se refere à percepção do suporte familiar das crianças participantes da pesquisa, observou-se que a grande maioria delas percebeu o suporte familiar como baixo e 30\% o perceberam como médio, não tendo havido na amostra nenhum indicativo de alto suporte familiar. Vale ressaltar que o suporte familiar, distinto da estrutura familiar, engloba outras características, nem sempre palpáveis ou de fácil mensuração. A noção de suporte familiar adequado nem sempre depende de maneira exclusiva da estrutura familiar constituída, ou seja, o modelo social que a cultura menciona como ideal: pai, mãe e irmãos morando em um mesmo local com suas funções econômicas e familiares definidas (Baptista et al., 2001). 
Com o objetivo de verificar a relação entre percepção de suporte familiar e traços de personalidade, uma pesquisa com 346 universitários do interior de São Paulo, de idade média de 25 anos, constatou que indivíduos com adequada percepção do suporte familiar apresentavam traços de personalidade adequados, como boa socialização, simpatia, responsabilidade, honestidade, capacidade de flexibilidade, maturidade, entre outros, enquanto os que apresentavam uma percepção negativa do suporte familiar demonstravam ansiedade, instabilidade emocional, imprevisibilidade, tendência a sentimentos confusos, dentre outros (Santos, 2006).

Em um estudo exploratório das propriedades psicométricas de uma escala de suporte familiar (FSS - Family Support Scale), identificou-se que o adequado suporte social e familiar pode ter efeito benéfico quando os indivíduos estão diante de situações de stress (Hanley, Tassé, Aman \& Prace, 1998).

Em relação à presença de stress, observou-se que, das 30 crianças avaliadas, 73,3\% apresentaram indicativo de stress caracterizado pelos sintomas de medo, alteração de apetite, aflição, tristeza e dificuldade para dormir. O stress pode ser reflexo de causas ou fontes externas ou internas (Lipp et al., 1991). As causas internas são criadas pela própria criança, ou seja, dependem da forma como a criança interpreta e reage diante de situações do seu dia a dia, de seus pensamentos (cognição) e do tipo de personalidade. É importante pensarmos nos eventos da vida que podem estar contribuindo para a presença de stress nessas crianças. Fatores como responsabilidade excessiva, problemas de saúde na família, problemas financeiros, nascimento de irmãos, separação dos pais, mudança de escola ou residência podem favorecer o stress nessa população (Lipp \& Novaes, 1998).

Dessa forma, as famílias podem vivenciar situações estressantes particulares, comumente referidas como mudanças de vida e eventos importantes, que podem desencadear repercussões sobre a saúde; nesse momento, a obtenção de suporte social desempenha um papel de destaque. Diferentes dimensões de suporte podem prevenir essa ruptura ou moderar o efeito negativo de situações percebidas como estressantes, protegendo as crianças contra a incerteza, cuidados precários, violência e isolamento (Mota, Lins, Franco \& Motta, 1999).
Estudos revelam que o stress excessivo pode levar a desordens psíquicas severas e apontam ainda o aparecimento de distúrbios comportamentais e psicológicos, tais como delinquência, abuso de drogas, suicídio, depressão, alteração do humor, alteração na autoestima e no desempenho, hiperatividade, agressividade, impulsividade e apatia, associados a situações desgastantes para a criança (Lipp \& Romano, 1987; Lipp et al., 1991).

Pesquisas também apontam associação entre adversidades afetivas na infância (consideradas como estressoras) e o desenvolvimento, na adolescência e na vida adulta, de neuroses, incluindo déficit de afeto positivo, instabilidades emocionais, falta de confiança, alterações cognitivas e psicopatologias, principalmente depressão, ansiedade, uso de drogas e álcool (Higley \& Suomi, 1996).

Foi possível constatar que $60,0 \%$ das crianças que viviam em famílias intactas apresentaram uma percepção de baixo suporte oferecido pelos membros da família e sintomas indicativos de stress. Já em relação às crianças que moravam em famílias uniparentais, 55,6\% apresentavam percepção de baixo suporte familiar, com sintomas de stress. Verifica-se, assim, que a estrutura familiar não constituiu, neste estudo, fator protetor contra o surgimento de sintomas de stress nem melhorou a percepção da criança quanto ao suporte familiar recebido. Ademais, famílias do tipo monoparental (com a presença de apenas um dos genitores) contribuíram de forma acentuada para a apresentação de sintomas de stress e também para uma baixa avaliação do suporte oferecido pela família.

Em geral, o comprometimento do bem-estar dos pais dificulta um bom funcionamento do sistema de suporte familiar. As alterações emocionais dos pais, conflitos familiares e infelicidades conjugais, entre outros fatores, interferem negativamente na qualidade afetiva dos relacionamentos familiares e, particularmente, no relacionamento entre seus membros.

Algumas limitações do estudo devem ser destacadas. A primeira está relacionada ao fato de o grupo estudado estar restrito às crianças participantes do grupo terapêutico de uma única unidade de saúde; dessa forma, os índices de stress podem ter sido subestimados e os dados finais podem apresentar distorções. A segunda limitação diz respeito à possibilidade de interferência do tipo de funcionamento da família nos resultados 
apresentados, variável não avaliada neste estudo. Contudo, pode-se pensar que, independentemente da configuração familiar da criança, é na qualidade do relacionamento entre os membros da sua família que recai a maior ou menor possibilidade de bem-estar. Por outro lado, alguns aspectos abordados poderão constituir indicadores importantes para o entendimento do stress infantil e do papel mediador do suporte familiar. Os resultados desta pesquisa sugerem a necessidade de estudos que explorem estratégias familiares eficazes no suporte dado à criança, tendo em vista a redução dos níveis de stress apresentados.

\section{Considerações Finais}

Há amplas evidências de que problemas relacionados à estrutura, ao funcionamento e ao suporte familiar estão relacionados a desordens psiquiátricas infantis, especificamente a presença de sintomas de stress, com consequentes alterações do estado emocional da criança.

As rápidas mudanças sociais e, principalmente, os novos padrões de organização familiar que estão sendo observados atualmente parecem ter alguma relação com a prevalência de alterações emocionais e com o comprometimento no desenvolvimento mental saudável da criança. Obviamente, não seriam somente esses fatores que contribuiriam para isso, porém não deve ser desprezado o fato de que a família funciona como um "colchão amortecedor" para os eventos estressores enfrentados pela criança no decorrer de seu processo de desenvolvimento.

Os resultados da pesquisa demonstraram que a presença de stress tem relação com a percepção, por parte da criança, de um suporte familiar inadequado, que está correlacionado negativamente com a presença de sintomas de stress nesta amostra. Tomando como base os resultados dos achados e das pesquisas acerca do papel do suporte social e do stress infantil, pode-se considerar o suporte familiar como um dos mais relevantes amortecedores do efeito de eventos estressores na vida da criança. Assim, conhecer a percepção de suporte familiar pode ser uma ferramenta importante no planejamento e na intervenção junto às famílias, uma vez que o suporte familiar tem efeito protetor sobre
Dessa forma, programas de prevenção do stress que utilizem a família como aliada poderão ser uma estratégia que favoreça um melhor suporte familiar e, consequentemente, um adequado enfrentamento dos estressores diários por parte da criança, evitando o aparecimento de doenças e de dificuldades advindas do stress excessivo.

\section{Referências}

Arpini, D. M., \& Quintana, A. M. (2003). Identidade, família e relações sociais em adolescentes de grupos populares. Estudos de Psicologia (Campinas), 20 (1), 27-36. doi: 10.15 90/S0103-166X2010000300006.

Baptista, M. N. (2004). Psicoterapias cognitivo-comporta mental e cognitiva: aspectos teóricos e terapêuticos no manejo da depressão e suicídio. In M. N. Baptista. Suicídio e depressão: atualizações (pp.161-176). Rio de Janeiro: Guanabara-Koogan.

Baptista, M. N. (2005). Desenvolvimento do inventário de percepção do suporte familiar: estudos psicométricos preliminares. Psico-USF, 10 (1), 11-19.

Baptista, M. N., Baptista, A. S. D., \& Dias, R. R. (2001). Estrutura e suporte familiar como fatores de risco na depressão de adolescentes. Psicologia Ciência e Profissão, 21 (2), 5261.

Brasil. Ministério da Saúde. (1997) Comitê Nacional de Ética e Pesquisas em Seres Humanos. Resolução 196, de 10 de outubro de 1996: diretrizes e normas regulamentadoras de pesquisa envolvendo seres humanos. Brasília: Autor.

Bugental, D. B., \& Johnston, C. (2000). Parental and child cognitions in the context of the family. Annual Review Psychology, 51, 315-344.

Carvalho, I. M. M., \& Almeida, P. H. (2003). Família e proteção social. São Paulo em Perspectiva, 17 (2), 109-122.

Egeland, B., \& Kreutzer, C. (1991). Maternal stress on highrisk children. In E.M. Cummings, A.L. Greene \& K, Karraker. Life-Span developmental psychology: perspectives on stress and coping. Hillsdale, New Jersey: Lawrence Erlhaum Associates Publishers.

Gomide, P. I. C. (2003). Estilos parentais e comportamento anti-social. In A. Del Prette \& Z. Del Prette (Orgs.), Habilidades sociais, desenvolvimento e aprendizagem: questões conceituais, avaliação e intervenção. Campinas: Alínea.

Instituto Brasileiro de Geografia e Estatística. (2001). Síntese de indicadores sociais 2000. Rio de Janeiro: Autor.

Hanley, B., Tassé, M. J., Aman, M. G., \& Prace, P. (1998). Psychometric properties of the family support scale with head start families. Journal of Child and Family Studies, 7 (1), 69-77.

Higley, J. D., \& Suomi, S. J. (1996). Effect of reactivity and social competence on individual responses to severe stress in children: investifations using nonhuman primates. In R. Pfeffer (Ed.), Severe stress and mental 
disturbance in children. Washington: American Psychiatric Press.

Labrador, F. J., \& Crespo, M. (1994). Evalución del estrés. In B. R. Fernandez. Evaluación conductual hoy: un enfoque para el cambio en psicologia clínica y de la salud (pp. 484-529). Madrid: Ediciones Pirámide.

Lakey, B., \& Scoboria, A. (2005). The relative contribuition of trait and social influences to the links among perceived social suport, affect, and self-esteem. Journal of Personality, 73 (2), 361-388.

Leventhal, T., \& Brooks-Gunn, J. (2000). The neighborhoods they live in. The effects of neighborhood residence on child and adolescent outcomes. Psychological Bulletin, 26, 309-337.

Lipp, M. (2000). O estresse da criança e suas conseqüências. In M. Lipp (Org.), Crianças estressada: causas sintomas e soluções (pp.13-42). Campinas: Papirus.

Lipp, M. E. N., \& Lucarelli, M. D. M. (1998). Escala de estresse infantil: manual. São Paulo: Casa do Psicólogo.

Lipp, M. E. N., \& Novaes, L.E. (1998). O stress. São Paulo: Contexto.

Lipp, M. N., \& Romano, A. S. F. (1987). O estresse infantil. Estudos de Psicologia (Campinas), 4, 42-54.

Lipp, M. E. N., Souza, E. A. P., Romano, A. S. P. F., \& Covolan, M. A. (1991). Como enfrentar o estresse infantil. São Paulo: Ícone Editora.

Miller, R., \& Jeff-Darlington, Y. (2002). Who supports? The providers of social support to dual-parent families caring for young children. Journal of Community Psyshology, 30 (5), 461-473.
Mota, E. L. A., Lins A., Franco, S., \& Motta, M. C. (1999). Migração, estresse e fatores psicossociais na determinação da saúde da criança. Psicologia Reflexão e Crítica, 12 (1), 119-132.

Saric, Z. R., Rijavec, M., \& Zganec, A. B. (2001). The relation of parental practices ans self-conception to young adolescent problem behaviors and substance use. Nord Journal Psychiatry, 55 (3), 203-209.

Santos, T. M. M. (2006). Evidencia de validade entre percepção desuportefamiliare traços de personalidade. Dissertação de mestrado não-publicada. Programa de Pós-Graduação Stricto Sensu em Psicologia, Universidade São Francisco, Itatiba.

Sbaraini, C. R. \& Schermann, L. B. (2008). Prevalence of childhood stress and associated factors: a study of schoolchildren in a city in Rio Grande do Sul Sate, Brazil. Cadernos de Saúde Pública, 24 (5), 1082-1088.

Tanganelli, M. S. L., \& Lipp, M. E. N. (1998). Sintomas de estresse na rede pública de ensino. Estudos de Psicologia (Campinas), 15 (3), 17-27.

Villela, M. V. (1996). Sintomas e fontes de stress em escolares de primeira a quarta séries. In M. Lipp (Org.), Pesquisas sobre stress no Brasil. Campinas: Papirus.

Wagner, A., Ribeiro, L., Arteche, A. X., \& Bornholdt, E. A. (1999). Configuração familiar e o bem-estar psicológico dos adolescentes. Psicologia Reflexão e Crítica, 12 (1), 147-156.

Recebido em: 19/11/2009

Versão final reapresentada em: 19/7/2010

Aprovado em: 11/3/2011 
